\title{
Custos correntes versus custos históricos no cálculo da taxa de lucro: uma proposta para solução empírica
}

Current costs versus historical costs in the rate of profit calculation: a proposal for empirical solution

\author{
Leonel Toshio Clemente ${ }^{\text {a }}$ \\ Eduardo Augusto de Lima Maldonado Filho ${ }^{b}$
}

\begin{abstract}
Resumo: Há extenso e controverso debate entre marxistas sobre como computar o capital fixo no denominador da taxa de lucro geral. Este artigo trata de duas formas de mensurar o capital fixo: a custos correntes e a custos históricos. O objetivo é propor uma solução empírica para este dilema. Para tanto, aplica-se um modelo de mudanças de regime Markoviano para a relação entre taxa de lucro e crescimento econômico dos EUA entre 1947 e 2007 e, para alguns períodos da série, aplicam-se correções na taxa de lucro a custos históricos a partir das variações da taxa de lucro a custos correntes. Constatou-se que a taxa de lucro a custos históricos corrigida apresentou maior capacidade de explicar o crescimento econômico do que as séries convencionais a custos históricos ou a custos correntes.
\end{abstract}

Palavras-chave: Taxa de Lucro; Marxismo; Capital Fixo; Mudança de Regime Markoviano Classificação JEL: B24, C18, P16

\begin{abstract}
There is extensive and controversial debate among Marxists about how to compute fixed capital in the denominator of the general rate of profit. This paper deals with two ways of measuring fixed capital: at current costs and at historical costs. The objective is to propose an empirical solution to this dilemma. For this purpose, a Markovian regime-switching model is applied to the relationship between the rate of profit and economic growth in the USA between 1947 and 2007 and corrections are applied for some periods of the series to the rate of profit at historical costs from changes in the rate of profit at current costs. It was found that the corrected rate of profit at historical costs was more capable of explaining economic growth than conventional series at historical costs or at current costs.
\end{abstract}

Keywords: Profit Rate; Marxism; Fixed Capital; Markovian-switching Regime

JEL Classification: B24, C18, P16

\footnotetext{
${ }^{\text {a }}$ Professor Adjunto da Faculdade de Ciências Econômicas da Universidade Federal do Rio Grande do Sul (FCE/UFRGS). Email: leonel_t_clemente@ hotmail.com.

${ }^{\mathrm{b}}$ Professor Aposentado da Faculdade de Ciências Econômicas e do Programa de Pós-Graduação em Economia da Universidade Federal do Rio Grande do Sul (FCE e PPGE/UFRGS). Email: emaldonadof@ gmail.com.
} 


\section{Introdução}

Para estimar dados de taxa de lucro média utilizando as contas nacionais, é necessário estimar uma proxy para o capital fixo, o qual é computado no denominador da taxa de lucro pela literatura marxista. Existem diferentes metodologias de cálculo do capital fixo empregadas por economistas marxistas, as quais podem ser agrupadas em: custos correntes, custos históricos e custos em tempo de trabalho socialmente necessário. $\mathrm{O}$ presente artigo trata das metodologias empregando custos correntes e custos históricos.

O capital fixo a custos correntes é o montante investido aos preços atuais, ou seja, aos preços que seriam pagos em caso de necessidade de reposição do capital. Por isso, custos correntes são também denominados de custos de reposição. A abordagem de custos históricos, por outro lado, considera o valor investido em capital fixo no momento do investimento. Há um extenso e acalorado debate teórico entre marxistas, resumido em Kliman (2009), sobre como o capital fixo deve ser computado no denominador da taxa de lucro.

Este artigo tem por objetivo propor uma solução empírica para esse debate. Para tanto, este trabalho está divido em quatro seções além desta introdução. Na Seção 2 são apresentadas minuciosamente as definições de custos históricos e de custos correntes, destacando as vantagens e desvantagens de cada metodologia. Na Seção 3 é apresentada e discutida uma solução empírica para o dilema da escolha da metodologia de custos históricos ou custos correntes. São utilizados os dados de taxa de lucro anuais estimados por Kliman (2009) para os EUA entre 1947 e 2007. Por fim, na Seção 4, são apresentadas as principais conclusões.

\section{Custos Históricos vs. Custos Correntes na Taxa de Lucro}

Nesta seção são apresentadas e comparadas as duas formas de estimação de taxa de lucro: empregando custos históricos e custos correntes. A taxa de lucro a custos a históricos, expressa por Kliman (2009, p.11-15), é dada por:

$$
r_{t}^{h}=\frac{\pi_{t}}{C_{t}^{h}}=\frac{\pi_{t}}{\sum_{t=0}^{t-1} I_{t}}
$$

Onde: $r_{t}^{h}=$ Taxa de lucro a custos históricos no período $t$

$\pi=$ Massa de lucro do período $t$

$C_{t}^{h}=$ Estoque de capital fixo a custos históricos no período $t$

$I_{t}=$ Investimento no período $t$

Desta forma, no denominador da taxa de lucro a custos históricos estão computados os valores efetivamente pagos no passado, no momento da aquisição do capital fixo. No numerador, a massa de lucro está a preços correntes. 
A taxa de lucro a custos correntes, por outro lado, é calculada utilizando o índice de preços dos itens de capital fixo, que atualiza os valores destes itens. Assim, esta taxa é baseada no custo corrente, ou atual, do capital fixo.

$$
r_{t}^{c}=\frac{\frac{\pi_{t}}{F_{t}}}{C_{t}^{c}}=\frac{\pi_{t}}{F_{t} \sum_{t=0}^{t-1}\left(\frac{I_{t}}{F_{t}}\right)}
$$

Onde: $r_{t}^{c}=$ Taxa de lucro a custos correntes no período $\mathrm{t}$

$C_{t}^{c}=$ Estoque de capital fixo a custos correntes no período $\mathrm{t}$

$F_{t}=$ Índice de preços dos itens de capital fixo no período $\mathrm{t}$

$I_{t}=$ Investimento no período $t$

Como se pode observar, a diferença no cálculo da taxa de lucro a custos históricos e a custos de reposição é que essa última corrige seu denominador pelo índice de preços dos itens de capital fixo. Portanto, no denominador da taxa de lucro a custos de reposição não está o computado o que foi efetivamente pago pelo capitalista no ato do adiantamento do capital fixo, mas o que custaria para fazer o investimento no período atual $(t)$.

\subsection{Custos de Reposição vs. Custos Históricos}

Nesta subseção são discutidas potencialidades e limitações das metodologias da taxa de lucro a custos de reposição e a custos históricos. Para tanto são consideradas algumas variações hipotéticas de preços e são comparados os seus efeitos sobre os cálculos das taxas a custos históricos e a custos de reposição. Por fim, são considerados os argumentos de Jones (2012) e Kliman (1988) sobre o tema.

Se houvesse perfeita estabilidade de preços (inflação zero), como se pode ver pelas expressões apresentadas anteriormente, as taxas de lucro a custos de reposição ou a custos históricos seriam equivalentes. Por outro lado, havendo aumento geral dos preços de forma homogênea entre os períodos $t-1$ e $t$, os efeitos sobre as taxas de lucro estimadas a custos de reposição e custos históricos seriam respectivamente:

$$
\frac{\uparrow \pi_{t}}{\uparrow F_{t} \sum_{t=0}^{t-1}\left(\frac{I_{t}}{F_{t}}\right)} \therefore r_{t}^{c}
$$

e

$$
\frac{\uparrow \pi_{t}}{\sum_{t=0}^{t-1} I_{t}} \therefore \uparrow r_{t}^{h}
$$

Onde: $\uparrow=$ aumento

Portanto, em caso de um aumento homogêneo ou proporcional dos preços dos meios de produção e das mercadorias como um todo (abstraindo-se aqui a força de 
trabalho), haveria aumento da taxa de lucro a custos históricos, enquanto a taxa de lucro a custos correntes não variaria. Este comportamento da taxa de lucro a custos de reposição é desejável, uma vez que aumentos homogêneos nos preços não representam aumento da taxa de lucro.

Se houvesse um aumento desproporcional dos preços dos meios de produção entre os períodos $t-1$ e $t$, ceteris paribus, resultaria que as taxas de lucro estimadas a custos de reposição e custos históricos variariam respectivamente da seguinte forma:

$$
\frac{\pi_{t}}{\uparrow F_{t} \sum_{t=0}^{t-1}\left(\frac{I t}{F_{t}}\right)} \therefore \downarrow r_{t}^{c}
$$

$\mathrm{e}$

$$
\frac{\pi_{t}}{\sum_{t=0}^{t-1} I_{t}} \therefore r_{t}^{h}
$$

Onde: $\downarrow=$ redução

Ou seja, havendo aumento relativo nos custos de reposição do capital, a taxa de lucro a custos de reposição diminuiria, enquanto a taxa de lucro a custos históricos se manteria constante. Nesse aspecto o comportamento da taxa de lucro a custos históricos é preferível ao dos custos correntes.

Por fim, se houvesse um aumento no índice geral de preços, mas o índice de preço dos itens do capital constante se mantivesse totalmente inalterado entre o período $t-1 \mathrm{e}$ $t$, então ter-se-iam os seguintes efeitos:

$$
\begin{aligned}
& \frac{\uparrow \pi}{F_{t} \sum_{t=0}^{t-1}\left(\frac{I_{t}}{F_{t}}\right)} \therefore \uparrow r_{t}^{c} \\
& \frac{\uparrow \pi}{\sum_{t=0}^{t-1} I_{t}} \therefore \uparrow r_{t}^{h}
\end{aligned}
$$

Neste caso, ambas as taxas de lucro aumentariam. Esse fenômeno seria esperado, pois o preço de venda das mercadorias para consumo improdutivo aumentaria em relação aos custos dos investimentos em capital constante. Deve-se destacar que esta generalização é não necessariamente é observada na prática, pois os fatores que levam ao aumento do índice geral de preços num contexto de estabilidade dos preços dos itens do capital constante poderiam afetar a lucratividade do departamento produtor de capital constante. A discussão de lucratividade setorial foi abstraída no presente artigo.

Em períodos de crise econômica, como na crise atual, cujo gatilho foi a pandemia de COVID-19, pode haver desvalorização do capital. Nesse caso, somente a taxa de lucro a custos de reposição seria capaz de captar as desvalorizações generalizadas do capital, ou seja, seria capaz de captar a queima de capital. Por outro lado, em condições normais de aumentos regulares nos níveis gerais de preço, ou inflação, as empresas tendem a contabilizar o valor dos seus meios de produção de baixa liquidez, como imóveis, plantas 
industriais e máquinas, com base no custo histórico de aquisição. Então, neste caso, o uso de taxa de lucro a custos de reposição não representaria bem a rentabilidade média dos capitais das empresas.

Kliman (1988) demonstrou matematicamente a não validade do Teorema de Okishio utilizando o Temporal Single System Interpretation (TSSI). Em sua formulação, o autor demonstra que, supondo constante a inovação tecnológica poupadora de trabalho, o capital constante contabilizado a custos correntes tende a ser progressivamente menor do que o capital constante contabilizado a custos históricos (KLIMAN, 1988, p.286). Este progressivo afastamento dos resultados das estimações do capital constante a custos históricos e a custos de reposição dá origem à divergência na tendência na taxa de lucro utilizando estas duas formas de cálculo. Porém, o autor considera que há períodos de crise em que o valor do capital é atualizado pelos preços de reposição:

É verdade que o capital acaba sendo reavaliado de acordo com o custo de reproduzi-lo, e que a desvalorização do capital, portanto, tende a aumentar a taxa de lucro. A "resolução" da discrepância entre o custo de produção original e os custos correntes de reprodução, porém, ocorre por meio dos diversos mecanismos da crise, através do ajuste forçado dos antigos valores aos novos. (KLIMAN, 1988, p. 286, tradução própria) ${ }^{1}$.

Assim, Kliman (1988, p.286) mostra uma limitação da análise da rentabilidade a custos históricos: não captar desvalorizações do capital durante as crises. A partir da análise dos efeitos da desvalorização do capital sobre a taxa de lucro por Marx, pode-se inferir que a taxa de lucro a custos históricos não representa a rentabilidade da economia em períodos de desvalorização do capital. Para Marx (1991a, p. 358, tradução própria)²:

1 "It is true that capital does eventually become revalued according to the cost of reproducing it, and that capital devaluation therefore tends to raise the profit rate. The "resolution" of discrepancy between original production cost and current reproduction costs, however, takes place through the many mechanisms of crisis, through the forcible adjustment of old values to the new".

2 "The periodical devaluation of the existing capital, which is a means, immanent to the capitalist mode of production, for delaying the fall in the profit rate and accelerating the accumulation of capital value by the formation of new capital, disturbs the given conditions in which the circulation and reproduction process of capital takes place, and is therefore accompanied by sudden stoppages and crises in the production process". 
A desvalorização periódica do capital existente, que é um meio, imanente ao modo de produção capitalista, de retardar a queda da taxa de lucro e acelerar a acumulação do valor do capital pela formação de novo capital, perturba as condições dadas em que o o processo de circulação e reprodução do capital ocorre e, portanto, é acompanhado por paradas repentinas e crises no processo de produção.

Acerca da importância de computar desvalorizações do capital, Kliman (2009) argumenta a respeito de uma ideia-chave na teoria marxiana da crise econômica: a destruição do capital por meio de crises. Trata-se de uma destruição física, mas também dos valores econômicos. Porém, como se demonstrou algebricamente na última equação, a taxa de lucro a custos históricos não é capaz de captar recuperações no nível de rentabilidade em função da queima de capital ocasionada pelo aumento no índice geral de preços. Portanto, a afirmação de Kliman (2009) de que a causa da crise de 2008 é baixa rentabilidade, a qual resulta da não desvalorização do capital constante, não poderia ser feita a partir de uma análise da taxa de lucro a custos históricos, como o próprio autor faz. Jones (2014) afirma este problema:

Se os resultados teóricos do TSSI dependessem da avaliação do custo histórico, essa crítica seria um grande problema, pois deixaria o TSSI incapaz de reproduzir a conclusão de Marx de que as crises permitem que a taxa de lucro se recupere pela desvalorização do capital. (JONES, 2014, p. 58, tradução própria) ${ }^{3}$

[...] Kliman argumenta que durante as crises da década de 1970 e início de 1980, não foi desvalorizado ou destruído capital suficiente para permitir um novo boom importante como o que ocorreu após a Segunda Guerra Mundial. É por isso que ele argumenta a taxa de lucro ter permanecido persistentemente baixa. Mas, uma vez que a taxa de lucro que Kliman mede não é afetada pela destruição dos valores do capital da forma como Marx descreve, Kliman não pode apelar à lei de Marx para explicar a taxa de lucro persistentemente baixa. Se os valores de capital existentes tivessem sido destruídos pela queda dos preços dos ativos da maneira que Marx descreve, isso não afetaria a medida escolhida por Kliman para a taxa de lucro. (JONES, 2014, p. 61, tradução própria) ${ }^{4}$.

\footnotetext{
3 "If the theoretical results of the TSSI depended on historical cost valuation, this criticism would be a major problem, since it would leave the TSSI unable to reproduce Marx's conclusion that crises allow the rate of profit to recover by devaluing capital".

4 " [...] Kliman argues that during the crises of the 1970s and early 1980s, not enough capital was devalued or destroyed to allow for a major new boom like the one that occurred after WWII. This is why, he argues, the rate of profit remained persistently low. But since the rate of profit which Kliman measures is not affected by the destruction of capital values in the way Marx describes, Kliman cannot appeal to Marx's law to explain the persistently low rate of profit. If existing capital values had been destroyed by falling asset prices in the way that Marx describes, this would not affect Kliman's chosen measure of the rate of profit".
} 
Tendo em vista as limitações das estimações a custos históricos e a custos correntes, como estimar uma taxa de lucro que represente os verdadeiros valores para os capitalistas e ao mesmo tempo permita a desvalorização do capital durante as crises como fenômeno típico do capitalismo?

\section{Uma Proposta de Solução Empírica para o Problema da Mensuração da Taxa de Lucro a Custos Históricos e a Custos de Reposição: o Modelo de Mudança de Regime Markoviano}

Nesta seção é apresentada uma metodologia de cálculo da taxa de lucro que se propõe a solucionar empiricamente o dilema entre a avaliação do capital fixo a custos históricos e a custos correntes. Por fim, são analisados os resultados da aplicação da metodologia. Assume-se, com base na discussão anterior, que em alguns períodos a taxa de lucro a custos históricos rege a economia e em outros deixa de ser relevante, pois, nos períodos de crise, os capitalistas reavaliam seus ativos a preços de reposição. Para identificar os períodos em que a taxa de lucro a custos históricos não se relacionou (ou teve relação diferente da esperada pela teoria) com o a Variação Absoluta do Produto Nacional Bruto Americano nominal (GGNP), realizou-se uma regressão com Mudança de Regime Markoviano.

No Modelo de Mudança de Regime Markoviano permite-se que os coeficientes da regressão mudem no tempo, submetidos a dois ou mais regimes. Para estes regimes se associa uma função de probabilidade no tempo, a qual indica qual o regime mais provável em cada período. Para estimar o modelo, partiu-se da especificação de uma regressão simples com intercepto e utilizou-se como variável explicativa a taxa de lucro a custos históricos (a razão entre excedente operacional líquido ou net operating surplus e estoque líquido de ativos privados a custo corrente (Current-Cost Net Stock of Private Fixed Assets, em inglês) com estoques obtidos em BEA (2015), calculada por Kliman (2009), denominada aqui como RH4.

A variável explicada é a variação absoluta do Produto Nacional Bruto Americano (GGNP). Foram permitidos dois regimes, definidos teoricamente como: períodos em que a taxa de lucro a custos histórico explica bem o GGNP (período de não crise) e períodos em que não explica bem (período de crise). O critério para diferenciar os dois regimes como crise ou não crise é a comparação entre os coeficientes estimados nos dois regimes: o maior coeficiente positivo estimado irá se referir ao período de não crise.

Especificou-se a restrição de que os regimes fazem variar apenas o coeficiente angular da regressão entre RH4 e GGNP, e não o coeficiente linear (intercepto) da regressão. Esta restrição foi aplicada porque a variação do coeficiente linear poderia afetar a interpretação da variação da relação entre RH4 e GGNP. Portanto, estimou-se utilizando o software PCGIVE (2016)um único coeficiente linear para todo o período dos dados, 1949 a 2009. Foram obtidos os seguintes coeficientes angulares: 


\section{Tabela 1 - Coeficientes Angulares Estimados}

\begin{tabular}{|c|c|c|c|}
\hline & \multirow{2}{*}{\multicolumn{2}{|c|}{ Coefficient }} & \multirow[b]{2}{*}{ t-prob } \\
\hline & & & \\
\hline & Constant & -5.79297 & 0.083 \\
\hline Regime R0 & RH4 (0) & 22.1517 & 0.100 \\
\hline Regime R1 & RH4 (1) & 42.6641 & 0.003 \\
\hline
\end{tabular}

Fonte: Elaboração própria.

Como esperado, existem dois regimes prováveis para a relação entre a taxa de lucro a custos históricos e o crescimento do produto nacional bruto americano. Em ambos regimes, como o esperado teoricamente, os coeficientes estimados são positivos. Sob o Regime R0 o coeficiente angular estimado é menor do que o estimado para o Regime R1, e não é significativo.. Por outro lado, o sob o Regime R1, o coeficiente é altamente significativo. Para verificar a confiabilidade destes testes de significância, aplicou-se o teste de normalidade de resíduo baseado em Doornik e Hansen (1994), estimado pelo PCGIVE (2016), e rejeitou-se a hipótese nula de normalidade do resíduo ao nível de significância de $5 \%$. Portanto, é pouco confiável a realização de testes de hipóteses utilizando a estatística $\mathrm{t}$ de Student.

Ainda assim, há indícios de que, sob o Regime R0, a variável RH4 ou não afeta, ou afeta menos GGNP do que sob o Regime R1. Portanto, considera-se que o Regime R0 representa períodos em que a RH4 explica mal a GGNP, ou seja, em R0 a economia não está sendo regida pela taxa de lucro a custos históricos, pois o capital fixo está sendo reavaliado. O regime R0 está provavelmente relacionado aos períodos de crise econômica. Por outro lado, sob o Regime R1, a taxa de lucro a custos históricos (RH4) explica bem o crescimento (GGNP), ou seja, em R1 a taxa de lucro a custos históricos rege a economia. Este Regime R1 é considerado o período de "normalidade" no processo de acumulação, período de não crise. $\mathrm{O}$ modelo estimado obteve convergência forte e, pela análise da função de autocorrelação de resíduos, considera-se que estes se comportam como ruído branco (white noise) . $^{5}$

Para visualizar os períodos de vigência dos regimes e suas probabilidades de ocorrência, pode-se visualizar a Tabela 2:

\footnotetext{
${ }^{5}$ Para detalhes dos dados e dos resultados estimados pode-se visualizar a saída do software Oxmetrics, pacote PCgive (2016), no Apêndice.
} 
Tabela 2 - Regimes e Probabilidades de Ocorrência no Tempo

\begin{tabular}{l|l|l}
\hline \hline Regime R0 & Período & Probabilidade média \\
\hline $1950-1950$ & 1 & 1.000 \\
\hline $1955-1955$ & 1 & 0.997 \\
\hline $1959-1959$ & 1 & 0.995 \\
\hline $1971-1971$ & 1 & 0.928 \\
\hline $1975-1976$ & 2 & 0.976 \\
\hline $1981-1981$ & 1 & 0.973 \\
\hline $1983-1983$ & 1 & 0.990 \\
\hline \hline
\end{tabular}

\begin{tabular}{l|l|l}
\hline \hline Regime R1 & Período & Probabilidade média \\
\hline $1949-1949$ & 1 & 1.000 \\
\hline $1951-1954$ & 4 & 1.000 \\
\hline $1956-1958$ & 3 & 0.983 \\
\hline $1960-1970$ & 11 & 0.988 \\
\hline $1972-1974$ & 3 & 0.996 \\
\hline $1977-1980$ & 4 & 0.997 \\
\hline $1982-1982$ & 1 & 0.993 \\
\hline \hline $1984-1991$ & 8 & 0.984 \\
\hline \hline
\end{tabular}

Fonte: Elaboração própria. 
Alternativamente à Tabela 2, para visualizar o comportamento da função de probabilidade de estar no regime R0 pode-se analisar o Gráfico 1:

\section{Gráfico 1 - Função de Probabilidade de Estar em Ro}

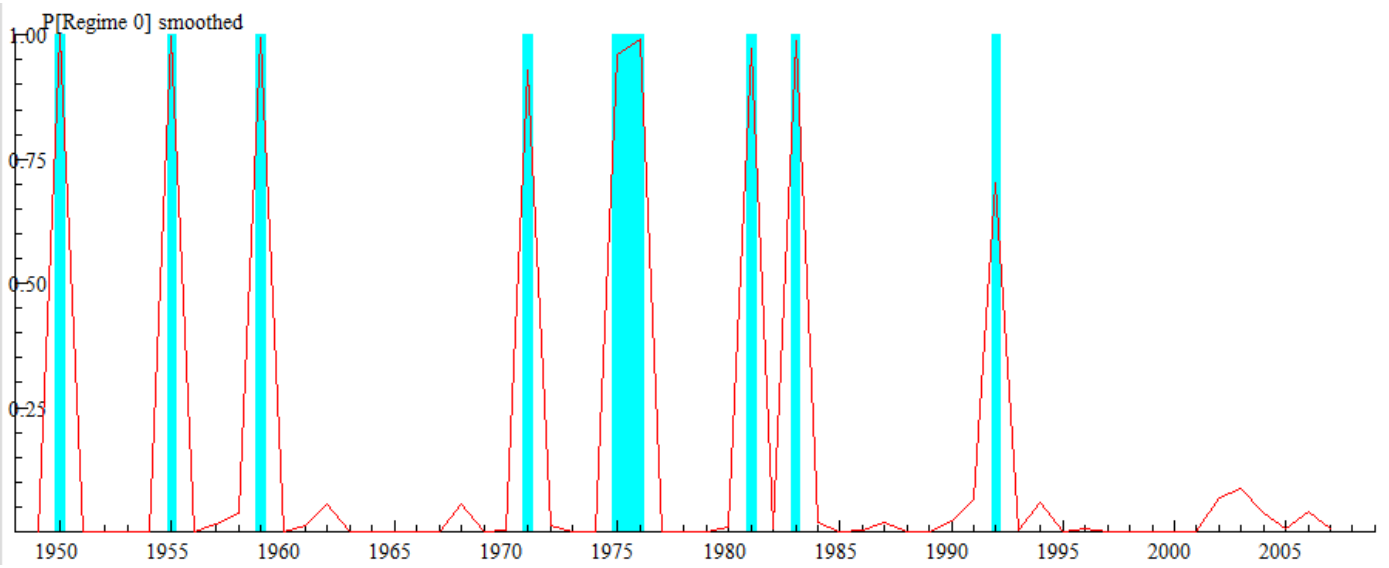

Fonte: Elaboração própria.

Do ponto de vista econométrico, o comportamento da função de probabilidade temporal de ocorrência dos regimes apresenta um bom resultado: a existência de valores extremos entre 0 e 1 indica que os regimes foram identificados com clareza.

Os períodos de recessão na economia norte-americana, publicados por publicados em Hamilton (2016) a partir do mesmo tipo de Modelo de Mudança de Regime Markoviano, são semelhantes aos períodos de crise identificados como Regime R0 no presente trabalho, como se pode visualizar no Gráfico 2. Os dados utilizados por Hamilton (2016) são trimestrais e se iniciam em 1968 e terminam em 2014, enquanto os tratados neste artigo são anuais para o período entre 1949 e 2009.

\section{Gráfico 2 - Recessões Inferidas por Hamilton (2016)}

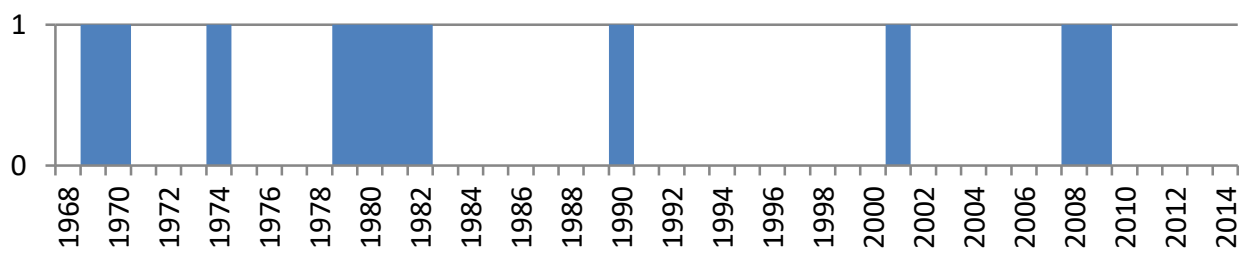

Fonte: Dados coletados em FRED (2016b) ${ }^{6}$ originários de Hamilton (2016).

\footnotetext{
${ }^{6}$ Os dados originais são trimestrais, então se utilizou como critério: se ocorreram dois ou mais trimestres de recessão durante o ano, considera-se ano de recessão.
} 
De modo geral, os períodos de vigência do Regime R0 ocorrem com atraso em relação às recessões identificadas por Hamilton (2016). A causa deste fenômeno pode estar relacionada a um atraso dos capitalistas no recálculo dos estoques de capital fixo. Durante as recessões os empresários não reconhecem desvalorizações do capital fixo imediatamente. Porém, depois de estabelecidos os novos preços médios, o capital é reavaliado. Este processo pode demorar alguns semestres ou anos segundo a comparação do Gráfico 1 com o Gráfico 2. Por exemplo, as recessões identificadas por Hamilton (2016) em 1969 e 1970 correspondem ao período de Regime R0 em 1970 apresentado no Gráfico 1. A recessão em 1974 apresentada no Gráfico 2 corresponde ao Regime R0 em 1975 e 1976 no Gráfico 1, a recessão entre 1979 e 1982 no Gráfico 2 corresponde ao Regime R0 em 1981 e 1983.

As funções probabilidade de recessão estimadas por Piger (2016) também se relacionam analogamente aos períodos de Regime R0 identificados neste artigo. No Gráfico 3 estão apresentadas as funções de probabilidade estimadas neste artigo com as estimadas por Piger (2016).

\section{Gráfico 3 - Comparações de Funções de Probabilidade}

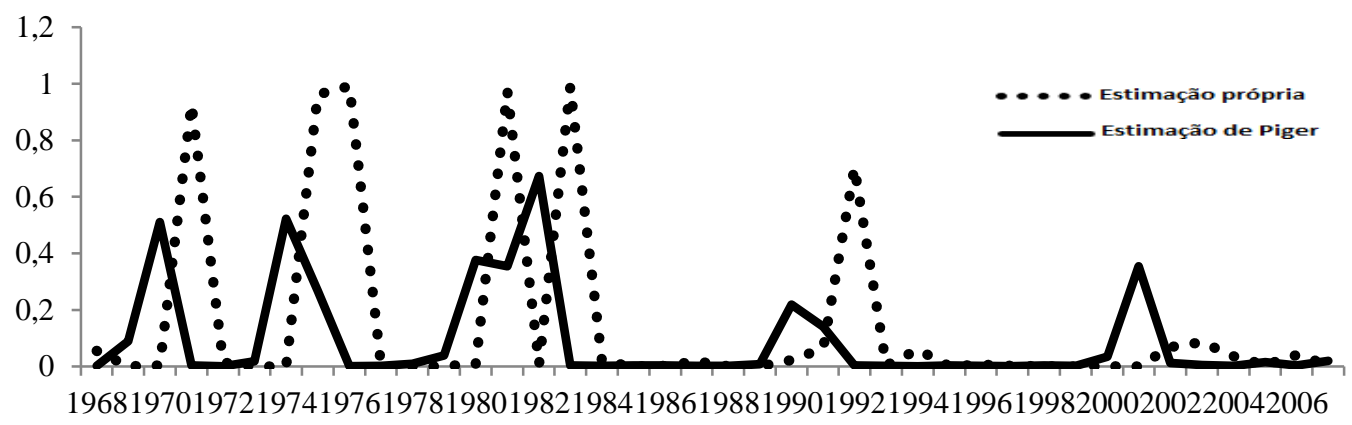

Fonte: Elaboração própria a partir dos dados estimados neste trabalho e dados de Piger (2016) ${ }^{7}$.

No Gráfico 3, a linha contínua representa a função de probabilidade de estar em recessão segundo Piger e em linha pontilhada está representada a função de probabilidade de estar sob o Regime R0, estimada neste artigo. Assim, como no Gráfico 2, pode-se constatar que a função de probabilidade de estar sob recessão segundo Piger (2016) antecipa os movimentos da função de Probabilidade de estar sob o Regime R0.

\footnotetext{
${ }^{7}$ Os dados de Piger (2016) são trimestrais. Para torna-los anuais, utilizou-se a média das probabilidades trimestrais ao longo do ano, o que removeu os picos de probabilidade e, por isso, a série tem poucos valores acima de 50\%. Não se deve utilizar esta série para interpretar se há ou não recessão. Aqui se utilizou apenas para ilustrar como os movimentos desta série de probabilidade antecipam os movimentos da série de probabilidade de Regime R0.
} 
Tendo em vista que a ocorrência do Regime R0 está associada com crises e reavaliação de ativos, é possível aprimorar os dados de taxa de lucro a custos correntes . Esse aprimoramento (que busca uma aproximação à taxa de lucro que os capitalistas consideram ao decidir sobre a acumulação de capital) deve combinar a série de taxa de lucro a custos históricos com a série a custos correntes. Considerando que as reavaliações de capital têm efeito permanente sobre a trajetória da taxa de lucro e não somente um efeito momentâneo, para realizar o aprimoramento da taxa de lucro fez-se necessário: transformar em taxa de variação anual a série de taxa de lucro a custos históricos (RH4) e, nos anos de Regime R0, substituir os dados de variações anual de RH4 por variações da taxa de lucro a custos de reposição (RC4).

A partir desta série híbrida de taxas de variação de taxa de lucro, tomando-se o primeiro dado de RH4 como verdadeiro, pode-se construir uma taxa de lucro a custos históricos corrigida pelas variações da taxa de lucro a custos correntes nos períodos de Regime R0. Essa taxa corrigida de lucro a custos históricos foi denominada RH**. Estas estimações estão apresentadas no Gráfico 4:

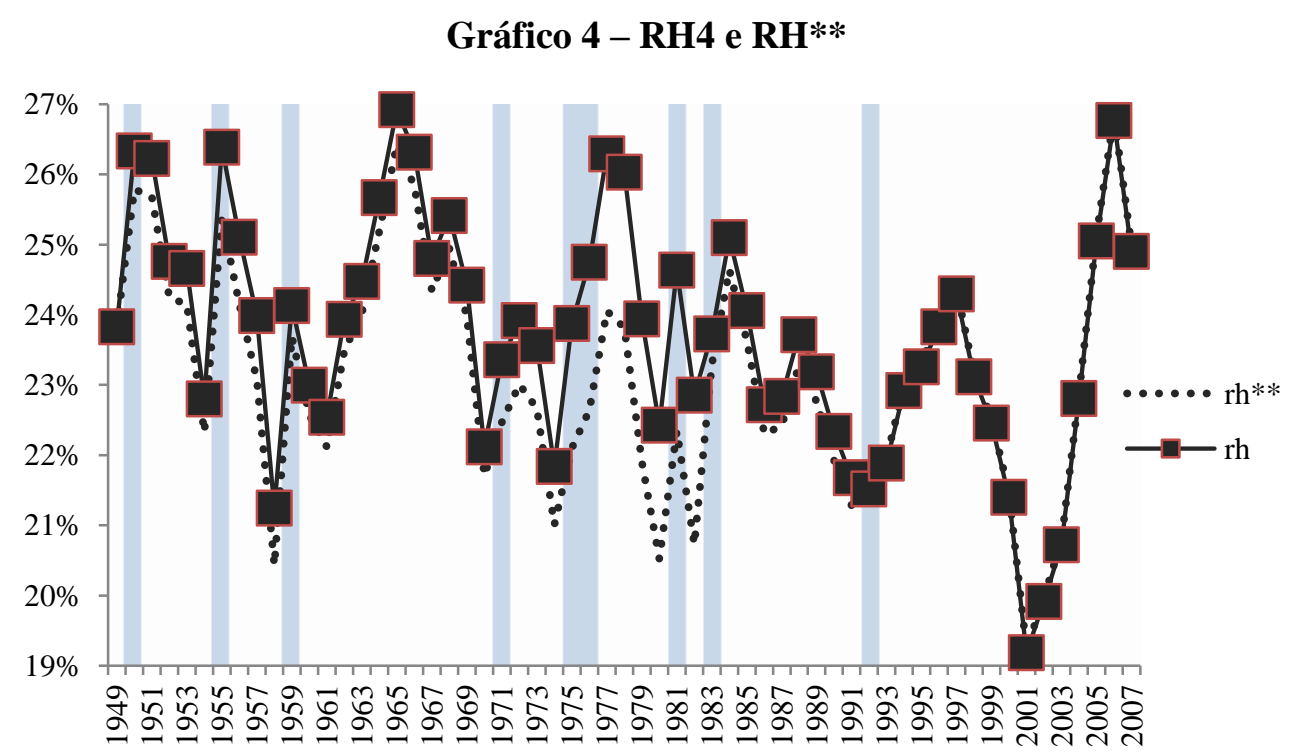

Fonte: Elaboração própria.

A partir do Gráfico 4, pode-se constatar que a série $\mathrm{RH}^{* *}$ tem valores persistentemente menores do que a série original RH4 desde 1950 até 1992, quando as séries $\mathrm{RH}^{* *}$ e RH4 convergem porque a taxa de lucro a custos correntes passam a variar em paralelo com a taxa RH4. Os dados estão apresentados em detalhes em Apêndice e pode-se notar que há aproximação entre $\mathrm{RH}^{* *}$ e RH sempre que a taxa de lucro a custos 
correntes tem movimentos paralelos à $\mathrm{RH}$, e há distanciamento entre $\mathrm{RH}^{*} *$ e RH sempre que a taxa de lucro a custos correntes tem movimentos divergentes à RH.

Esta série corrigida $\mathrm{RH}^{* *}$ foi utilizada no modelo de mudança de regimes com o GGNP, com o objetivo de comparar o comportamento dos regimes identificados com o modelo que utilizou a série original RH4 e GGNP. Assim como para a regressão com mudança de regime markoviano para RH4 e GGNP, foram identificados regimes semelhantes. Detalhes sobre a estimação do modelo podem ser visualizados no Apêndice. Testes de raiz unitária indicaram estacionariedade para $\mathrm{RH}^{* *}$ e $\mathrm{RH} 4$, assim como para GGNP. Regressões simples sem possibilidade de mudança de regime foram estimadas para comparar coeficientes de determinação e significância: o modelo com RH4 e GGNP não foi significativo a $5 \%$ de significância e obteve coeficiente de determinação de $3 \%$, enquanto o modelo que utilizou $\mathrm{RH}^{* *}$ foi significativo a $10 \%$ de significância e obteve coeficiente de determinação de $6 \%$.

Os modelos para explicar GGNP e $\mathrm{RH}^{* *}$ com mudança de regime identificaram regimes em períodos semelhantes e geraram coeficientes de mesmo sinal, níveis de significância próximos e coeficiente de determinação próximos. Possivelmente não é a queima de capital, ou desvalorização de capital durante as recessões, que causa o padrão identificado como Regime R0. Para verificar esta hipótese se estimou um modelo de mudança de regime Markoviano para RC4 e GGNP e, como se pode ver no Apêndice, foram identificados regimes semelhantes, ainda que menos frequentes ${ }^{8}$ do que os regimes Regime R0 do modelo de RH4 e GGNP. Qual a explicação da existência de Regime R0 no modelo de RH4 e GGNP em períodos em que não foi identificado regime semelhante no modelo de RC4 e GGNP? Qual a explicação da existência de Regime R0 no modelo de RH4 e GGNP em períodos em que também foi identificado Regime R0 no modelo de RC4 e GGNP? Para a primeira pergunta, a resposta está na diferença entre as metodologias de estimação da taxa de lucro RC4 e RH4. O fato de RH4 não permitir desvalorização do capital em recessões faz com que em alguns períodos RH4 esteja sob o Regime R0, enquanto RC4, em seu modelo, não esteja. Para a segunda pergunta, existem duas possíveis explicações que não são mutuamente excludentes: a natureza das séries faz com que a taxa de lucro, tanto a custos históricos ou a custos de reposição, tenha dois padrões de relação com a variação absoluta do produto (GGNP).

Esta resposta poderia ter respaldo na concepção de a taxa de lucro do período atual representar a variação absoluta máxima do produto no período seguinte. Ou seja, poderia haver dois padrões: períodos em que o crescimento se realizada próximo à taxa de lucro e

\footnotetext{
${ }^{8} \mathrm{O}$ fato de haver menor frequência de regimes R0 na regressão de RC4 e GGNP do que RH4 e GGNP significa que a relação entre RC4 e GGNP é mais constante do que RH4 com GGNP. Isso, do ponto de vista de previsão de GGNP, é um aspecto positivo de RC4 em relação a RH4. Por outro lado, para previsão de recessões, tendo em vista que a função de probabilidade de R0 da regressão de RH4 contra GGNP está altamente correlacionada com a função de probabilidade de recessão estimada por Piger (2016), a variável RH4 tem maior capacidade de prever recessões a partir das previsões de probabilidade de entrar no Regime R0. Em suma, comparando com a relação entre RC4 e GGNP, o aspecto negativo de inconstância relativa na relação entre RH4 e GGNP tem o aspecto positivo de proporcionar uma maior capacidade de previsão de recessões.
} 
períodos em que isso não ocorre, por exemplo. Outra explicação poderia ser o tempo de rotação do capital, que muda bruscamente em períodos de recessão. Tendo em vista que as taxas de lucro estimadas como RC4 e RH4 não levam em conta o tempo de rotação do capital, isso pode causar dois padrões de relação entre a taxa de lucro e o crescimento do produto.

A partir do Gráfico 4, pode-se constatar que a série $\mathrm{RH}^{* *}$ tem valores persistentemente menores do que a série original RH4 desde 1950 até 1992, quando as séries $\mathrm{RH}^{* *}$ e $\mathrm{RH} 4$ convergem porque a taxa de lucro a custos correntes passam a variar em paralelo com a taxa RH4. Os dados estão apresentados em detalhes em Apêndice e pode-se notar que há aproximação entre $\mathrm{RH}^{* *}$ e $\mathrm{RH}$ sempre que a taxa de lucro a custos correntes tem movimentos paralelos à $\mathrm{RH}$, e há distanciamento entre $\mathrm{RH}^{*} *$ e $\mathrm{RH}$ sempre que a taxa de lucro a custos correntes tem movimentos divergentes à $\mathrm{RH}$.

Esta série corrigida $\mathrm{RH}^{* *}$ foi utilizada no modelo de mudança de regimes com o GGNP, com o objetivo de comparar o comportamento dos regimes identificados com o modelo que utilizou a série original RH4 e GGNP. Assim como para a regressão com mudança de regime markoviano para RH4 e GGNP, foram identificados regimes semelhantes. Detalhes sobre a estimação do modelo podem ser visualizados no Apêndice. Testes de raiz unitária indicaram estacionariedade para $\mathrm{RH}^{* *}$ e $\mathrm{RH} 4$, assim como para GGNP.. Regressões simples sem possibilidade de mudança de regime foram estimadas para comparar coeficientes de determinação e significância: o modelo com RH4 e GGNP não foi significativo a $5 \%$ de significância e obteve coeficiente de determinação de 3\%, enquanto o modelo que utilizou $\mathrm{RH}^{* *}$ foi significativo a $10 \%$ de significância e obteve coeficiente de determinação de $6 \%$.

Os modelos para explicar GGNP e $\mathrm{RH}^{* *}$ com mudança de regime identificaram regimes em períodos semelhantes e geraram coeficientes de mesmo sinal, níveis de significância próximos e coeficiente de determinação próximos. Possivelmente não é a queima de capital, ou desvalorização de capital durante as recessões, que causa o padrão identificado como Regime R0. Para verificar esta hipótese se estimou um modelo de mudança de regime Markoviano para RC4 e GGNP e, como se pode ver no Apêndice, foram identificados regimes semelhantes, ainda que menos frequentes do que os regimes Regime R0 do modelo de RH4 e GGNP.

Qual a explicação da existência de Regime R0 no modelo de RH4 e GGNP em períodos em que não foi identificado regime semelhante no modelo de RC4 e GGNP? Qual a explicação da existência de Regime R0 no modelo de RH4 e GGNP em períodos em que também foi identificado Regime R0 no modelo de RC4 e GGNP? Para a primeira pergunta, a resposta está na diferença entre as metodologias de estimação da taxa de lucro RC4 e RH4.

O fato de RH4 não permitir desvalorização do capital em recessões faz com que em alguns períodos RH4 esteja sob o Regime R0, enquanto RC4, em seu modelo, não esteja. Para a segunda pergunta, existem duas possíveis explicações que não são mutuamente excludentes: a natureza das séries faz com que a taxa de lucro, tanto a custos 
históricos ou a custos de reposição, tenha dois padrões de relação com a variação absoluta do produto (GGNP) ${ }^{9}$.

Esta resposta poderia ter respaldo na concepção de a taxa de lucro do período atual representar a variação absoluta máxima do produto no período seguinte. Ou seja, poderia haver dois padrões: períodos em que o crescimento se realizada próximo à taxa de lucro e períodos em que isso não ocorre, por exemplo. Outra explicação poderia ser o tempo de rotação do capital, que muda bruscamente em períodos de recessão. Tendo em vista que as taxas de lucro estimadas como RC4 e RH4 não levam em conta o tempo de rotação do capital, isso pode causar dois padrões de relação entre a taxa de lucro e o crescimento do produto.

\section{Conclusão}

Neste artigo desenvolveu-se uma proposta metodológica de solução empírica para o problema de como computar os dados de capital fixo no denominador da taxa de lucro. Para tanto, realizou-se, a partir de dados de Kliman (2009), um modelo de mudança de regime Markoviano para identificar períodos em que a taxa de lucro a custos históricos não rege a economia. Nestes períodos, que coincidiram com períodos após recessões econômicas nos EUA, foram utilizadas variações da taxa de lucro a custos correntes para corrigir as variações da taxa de lucro a custos históricos. O resultado obtido em regressões simples foi que a taxa de lucro a custos históricos corrigida $\left(\mathrm{RH}^{* *}\right)$ apresentou maior coeficiente de determinação com o crescimento econômico (GGNP) do que as séries originais. No modelo com RH4 e GGNP se obteve coeficiente de determinação de 3\%, enquanto no modelo que utilizou $\mathrm{RH}^{* *}$ se obteve coeficiente de determinação de $6 \%$.

A partir de testes com a série corrigida também foram identificadas mudanças de regime. Este fato, inesperado, deu espaço para questionamentos: Por que alguns regimes não foram identificados com a série corrigida? Porque alguns regimes identificados anteriormente com a série original persistiram com a série corrigida? Para a primeira questão considera-se que de fato os capitalistas reavaliam seus investimentos no período de crise e assim a taxa de lucro corrigida ( $\left.\mathrm{RH}^{* *}\right)$ tem uma relação mais estável com o crescimento (GGNP) do que a taxa de lucro original (RH4). A resposta para a segunda pergunta pode estar relacionada às variações do tempo de rotação do capital, as quais não foram incorporadas na série de taxa de lucro, ou à própria natureza da lucratividade.

\footnotetext{
${ }^{9} \mathrm{O}$ fato de haver menor frequência de regimes R0 na regressão de RC4 e GGNP do que RH4 e GGNP significa que a relação entre RC4 e GGNP é mais constante do que RH4 com GGNP. Isso, do ponto de vista de previsão de GGNP, é um aspecto positivo de RC4 em relação a RH4. Por outro lado, para previsão de recessões, tendo em vista que a função de probabilidade de R0 da regressão de RH4 contra GGNP está altamente correlacionada com a função de probabilidade de recessão estimada por Piger (2016), a variável RH4 tem maior capacidade de prever recessões a partir das previsões de probabilidade de entrar no Regime R0. Em suma, comparando com a relação entre RC4 e GGNP, o aspecto negativo de inconstância relativa na relação entre RH4 e GGNP tem o aspecto positivo de proporcionar uma maior capacidade de previsão de recessões.
} 


\section{Referências}

DOORNIK, J. A. HANSEN H. A practical test for univariate and multivariate normality.

Discussion Paper, Nuffield College. 1994. Disponível em:

http://www.doornik.com/research/normal2.pdf.

FRED. Dates of U.S. recessions as inferred by GDP: based recession indicator. Federal Reserve Bank of St. Louis. Disponível em https://research.stlouisfed.org/fred2/series/JHDUSRGDPBR.

HAMILTON, J. D. The Econbrowser Recession Indicator Index. Disponível em: http://econbrowser.com/recession-index.

JONES, P. Depreciation, Devaluation and the Rate of Profit. 2012. Disponível em: http://thenextrecession.files.wordpress.com/2012/07/jones_peterdepreciation_devaluation_and_the_rate_of_profit_final.pdf.

KLIMAN, A. The Persistent Fall in Profitability Underlying the Current Crisis: New Temporalist Evidence. 2009. Disponível em:

http://akliman.squarespace.com/storage/Persistent\%20Fall\%20whole\%20primo\%2010.17 .09.pdf.

KLIMAN, A. The Profit Rate Under Continuous Technological Change. Review of Radical Political Economics, 20, no. 2-3 (June 1, 1988): 283-89. DOI: https://doi.org/10.1177/048661348802000243

KLIMAN, A. The Failure of the Capitalist Production: Underlying Causes of the Great Recession. Pluto Press, 2011.

MARX, K. Capital. The Process of Capitalist Production as a Whole.V.3. Penguin Books. New York: Clays Ltd, 1991.

PCGIVE. OxMetrics 7. Disponível em: < http://www.doornik.com/pcgive/>.

PIGER, J. Smoothed U.S. Recession Probabilities. Disponível em: http://pages.uoregon.edu/jpiger/us_recession_probs.htm/. 


\section{Apêndice}

\section{Dados de taxa de lucro para os EUA a custos históricos corrigidos pelas variações da taxa de lucro a custos correntes nos regimes 0 :}

\begin{tabular}{|c|c|c|c|c|c|c|c|c|c|c|}
\hline Ano & Re4 & Rh4 & $\mathbf{P}(\mathbf{R}=\mathbf{1})$ & $\mathbf{R}=\mathbf{1}$ & $\mathbf{R}=\mathbf{0}$ & rh* & rc4t/rc4t-1 & rht/rht-1 & $\begin{array}{c}\text { Variação de } \\
\text { rh }^{* *}\end{array}$ & $\mathbf{r h}^{* * *}$ \\
\hline 1949 & $14,8 \%$ & $23,8 \%$ & .99995936798684415 & 1 & 0 & $23,83 \%$ & 0,9768 & 0,9502 & 0,9502 & $23,83 \%$ \\
\hline 1950 & $16,0 \%$ & $26,3 \%$ & .0002593745813164205 & 0 & 1 & $25,79 \%$ & 1,0823 & 1,1052 & 1,0823 & $25,79 \%$ \\
\hline 1951 & $16,0 \%$ & $26,2 \%$ & .99999996134904978 & 1 & 0 & $26,23 \%$ & 1,0014 & 0,9960 & 0,9960 & $25,68 \%$ \\
\hline 1952 & $15,3 \%$ & $24,8 \%$ & .99999880725426715 & 1 & 0 & $24,76 \%$ & 0,9539 & 0,9440 & 0,9440 & $24,25 \%$ \\
\hline 1953 & $15,4 \%$ & $24,7 \%$ & .99848145439898983 & 1 & 0 & $24,66 \%$ & 1,0072 & 0,9959 & 0,9959 & $24,15 \%$ \\
\hline 1954 & $14,7 \%$ & $22,8 \%$ & .99958280812468847 & 1 & 0 & $22,80 \%$ & 0,9529 & 0,9246 & 0,9246 & $22,33 \%$ \\
\hline 1955 & $16,7 \%$ & $26,4 \%$ & .0028848253086286315 & 0 & 1 & $25,92 \%$ & 1,1369 & 1,1574 & 1,1369 & $25,38 \%$ \\
\hline 1956 & $15,3 \%$ & $25,1 \%$ & .99999889897513383 & 1 & 0 & $25,11 \%$ & 0,9157 & 0,9515 & 0,9515 & $24,15 \%$ \\
\hline 1957 & $14,6 \%$ & $24,0 \%$ & .98479399568747428 & 1 & 0 & $23,99 \%$ & 0,9539 & 0,9554 & 0,9554 & $23,07 \%$ \\
\hline 1958 & $13,5 \%$ & $21,2 \%$ & .96390254494369598 & 1 & 0 & $21,24 \%$ & 0,9265 & 0,8856 & 0,8856 & $20,43 \%$ \\
\hline 1959 & $15,7 \%$ & $24,1 \%$ & .0053539378750827793 & 0 & 1 & $24,62 \%$ & 1,1592 & 1,1361 & 1,1592 & $23,69 \%$ \\
\hline 1960 & $15,4 \%$ & $23,0 \%$ & .99999826375161127 & 1 & 0 & $23,00 \%$ & 0,9803 & 0,9528 & 0,9528 & $22,57 \%$ \\
\hline 1961 & $15,6 \%$ & $22,5 \%$ & .98837989917942715 & 1 & 0 & $22,54 \%$ & 1,0133 & 0,9801 & 0,9801 & $22,12 \%$ \\
\hline 1962 & $17,0 \%$ & $23,9 \%$ & .94340916916514717 & 1 & 0 & $23,93 \%$ & 1,0898 & 1,0619 & 1,0619 & $23,49 \%$ \\
\hline 1963 & $17,8 \%$ & $24,5 \%$ & .99996478947736234 & 1 & 0 & $24,48 \%$ & 1,0477 & 1,0227 & 1,0227 & $24,02 \%$ \\
\hline 1964 & $18,7 \%$ & $25,7 \%$ & .9984744681360338 & 1 & 0 & $25,67 \%$ & 1,0522 & 1,0486 & 1,0486 & $25,19 \%$ \\
\hline 1965 & $19,6 \%$ & $26,9 \%$ & .99966990753226281 & 1 & 0 & $26,92 \%$ & 1,0503 & 1,0489 & 1,0489 & $26,42 \%$ \\
\hline 1966 & $19,1 \%$ & $26,3 \%$ & .99996849052583237 & 1 & 0 & $26,32 \%$ & 0,9726 & 0,9775 & 0,9775 & $25,83 \%$ \\
\hline 1967 & $18,0 \%$ & $24,8 \%$ & .99995550567828584 & 1 & 0 & $24,80 \%$ & 0,9421 & 0,9424 & 0,9424 & $24,34 \%$ \\
\hline 1968 & $18,1 \%$ & $25,4 \%$ & .94457539237706134 & 1 & 0 & $25,41 \%$ & 1,0051 & 1,0247 & 1,0247 & $24,94 \%$ \\
\hline 1969 & $17,0 \%$ & $24,4 \%$ & .99967343122036934 & 1 & 0 & $24,42 \%$ & 0,9415 & 0,9611 & 0,9611 & $23,97 \%$ \\
\hline 1970 & $15,0 \%$ & $22,1 \%$ & .99709076447029321 & 1 & 0 & $22,12 \%$ & 0,8840 & 0,9057 & 0,9057 & $21,71 \%$ \\
\hline 1971 & $15,6 \%$ & $23,4 \%$ & .072157164011063604 & 0 & 1 & $22,89 \%$ & 1,0349 & 1,0559 & 1,0349 & $22,47 \%$ \\
\hline 1972 & $15,8 \%$ & $23,9 \%$ & .98671121379627369 & 1 & 0 & $23,92 \%$ & 1,0158 & 1,0240 & 1,0240 & $23,01 \%$ \\
\hline 1973 & $15,1 \%$ & $23,6 \%$ & .99993230052794868 & 1 & 0 & $23,56 \%$ & 0,9548 & 0,9852 & 0,9852 & $22,67 \%$ \\
\hline 1974 & $12,5 \%$ & $21,8 \%$ & .99998912930473072 & 1 & 0 & $21,84 \%$ & 0,8276 & 0,9269 & 0,9269 & $21,01 \%$ \\
\hline 1975 & $13,2 \%$ & $23,9 \%$ & .038497051707759904 & 0 & 1 & $22,98 \%$ & 1,0520 & 1,0930 & 1,0520 & $22,10 \%$ \\
\hline 1976 & $13,5 \%$ & $24,7 \%$ & .0085107182275566146 & 0 & 1 & $23,54 \%$ & 1,0243 & 1,0366 & 1,0243 & $22,64 \%$ \\
\hline 1977 & $14,0 \%$ & $26,3 \%$ & .99963907543856312 & 1 & 0 & $26,29 \%$ & 1,0381 & 1,0625 & 1,0625 & $24,05 \%$ \\
\hline 1978 & $13,6 \%$ & $26,0 \%$ & .99895511940825932 & 1 & 0 & $26,03 \%$ & 0,9723 & 0,9901 & 0,9901 & $23,82 \%$ \\
\hline 1979 & $12,1 \%$ & $23,9 \%$ & .99989932640037138 & 1 & 0 & $23,94 \%$ & 0,8923 & 0,9197 & 0,9197 & $21,90 \%$ \\
\hline 1980 & $11,0 \%$ & $22,4 \%$ & .98995885888725721 & 1 & 0 & $22,43 \%$ & 0,9054 & 0,9367 & 0,9367 & $20,52 \%$ \\
\hline 1981 & $12,0 \%$ & $24,6 \%$ & .026989910816144297 & 0 & 1 & $24,42 \%$ & 1,0889 & 1,0985 & 1,0889 & $22,34 \%$ \\
\hline 1982 & $11,4 \%$ & $22,9 \%$ & .99288349930654884 & 1 & 0 & $22,85 \%$ & 0,9546 & 0,9277 & 0,9277 & $20,73 \%$ \\
\hline 1983 & $12,8 \%$ & $23,7 \%$ & .010339092813951721 & 0 & 1 & $25,69 \%$ & 1,1239 & 1,0382 & 1,1239 & $23,29 \%$ \\
\hline 1984 & $14,2 \%$ & $25,1 \%$ & .98218204104297413 & 1 & 0 & $25,10 \%$ & 1,1063 & 1,0578 & 1,0578 & $24,64 \%$ \\
\hline 1985 & $14,1 \%$ & $24,1 \%$ & .99999930178093799 & 1 & 0 & $24,06 \%$ & 0,9924 & 0,9588 & 0,9588 & $23,62 \%$ \\
\hline 1986 & $13,7 \%$ & $22,7 \%$ & .99748555453023946 & 1 & 0 & $22,72 \%$ & 0,9743 & 0,9441 & 0,9441 & $22,30 \%$ \\
\hline 1987 & $14,1 \%$ & $22,8 \%$ & .98197248186610575 & 1 & 0 & $22,84 \%$ & 1,0284 & 1,0053 & 1,0053 & $22,42 \%$ \\
\hline 1988 & $14,7 \%$ & $23,7 \%$ & .99891796023646773 & 1 & 0 & $23,72 \%$ & 1,0398 & 1,0385 & 1,0385 & $23,28 \%$ \\
\hline 1989 & $14,4 \%$ & $23,2 \%$ & .99986325097445461 & 1 & 0 & $23,19 \%$ & 0,9792 & 0,9777 & 0,9777 & $22,77 \%$ \\
\hline 1990 & $13,9 \%$ & $22,3 \%$ & .97699245041173999 & 1 & 0 & $22,34 \%$ & 0,9651 & 0,9632 & 0,9632 & $21,93 \%$ \\
\hline 1991 & $13,9 \%$ & $21,7 \%$ & .93582521496601978 & 1 & 0 & $21,68 \%$ & 0,9985 & 0,9706 & 0,9706 & $21,28 \%$ \\
\hline 1992 & $14,0 \%$ & $21,5 \%$ & .29627689198190249 & 0 & 1 & $21,98 \%$ & 1,0140 & 0,9926 & 1,0140 & $21,58 \%$ \\
\hline 1993 & $14,3 \%$ & $21,9 \%$ & .99694080941459229 & 1 & 0 & $21,88 \%$ & 1,0185 & 1,0170 & 1,0170 & $21,95 \%$ \\
\hline 1994 & $15,0 \%$ & $22,9 \%$ & .94032287805267245 & 1 & 0 & $22,92 \%$ & 1,0513 & 1,0473 & 1,0473 & $22,99 \%$ \\
\hline 1995 & $15,3 \%$ & $23,3 \%$ & .99905302236684246 & 1 & 0 & $23,26 \%$ & 1,0169 & 1,0148 & 1,0148 & $23,33 \%$ \\
\hline 1996 & $15,9 \%$ & $23,8 \%$ & .9930898760579282 & 1 & 0 & $23,83 \%$ & 1,0430 & 1,0244 & 1,0244 & $23,90 \%$ \\
\hline 1997 & $16,5 \%$ & $24,3 \%$ & .99858979762414257 & 1 & 0 & $24,30 \%$ & 1,0348 & 1,0198 & 1,0198 & $24,37 \%$ \\
\hline 1998 & $15,9 \%$ & $23,1 \%$ & .99979271561463334 & 1 & 0 & $23,12 \%$ & 0,9664 & 0,9514 & 0,9514 & $23,19 \%$ \\
\hline 1999 & $15,7 \%$ & $22,5 \%$ & .9999034675115358 & 1 & 0 & $22,45 \%$ & 0,9829 & 0,9713 & 0,9713 & $22,52 \%$ \\
\hline 2000 & $14,9 \%$ & $21,4 \%$ & .99970604745477254 & 1 & 0 & $21,40 \%$ & 0,9523 & 0,9531 & 0,9531 & $21,46 \%$ \\
\hline 2001 & $13,6 \%$ & $19,2 \%$ & .99990542568095209 & 1 & 0 & $19,18 \%$ & 0,9090 & 0,8964 & 0,8964 & $19,24 \%$ \\
\hline 2002 & $14,4 \%$ & $19,9 \%$ & .9319334119936894 & 1 & 0 & $19,92 \%$ & 1,0611 & 1,0382 & 1,0382 & $19,98 \%$ \\
\hline 2003 & $14,9 \%$ & $20,7 \%$ & .91397677318042492 & 1 & 0 & $20,72 \%$ & 1,0379 & 1,0404 & 1,0404 & $20,78 \%$ \\
\hline 2004 & $15,7 \%$ & $22,8 \%$ & .96333415986889037 & 1 & 0 & $22,81 \%$ & 1,0527 & 1,1006 & 1,1006 & $22,87 \%$ \\
\hline 2005 & $16,4 \%$ & $25,1 \%$ & .99441067505251124 & 1 & 0 & $25,05 \%$ & 1,0403 & 1,0985 & 1,0985 & $25,13 \%$ \\
\hline 2006 & $16,9 \%$ & $26,8 \%$ & .95944979133494968 & 1 & 0 & $26,77 \%$ & 1,0340 & 1,0684 & 1,0684 & $26,85 \%$ \\
\hline 2007 & $15,7 \%$ & $24,9 \%$ & .99356443115914483 & 1 & 0 & $24,90 \%$ & 0,9293 & 0,9304 & 0,9304 & $24,98 \%$ \\
\hline
\end{tabular}

